\title{
EFFECT OF CULTIVAR AND GROWING LOCATION ON GINNING EFFICIENCY AND COTTON QUALITY
}

\author{
EL-FEKY, H.H. AND S.A. HASSAN
}

Cotton Research Institute, $A R C$, Giza

(Manuscript received 6 February 2011)

\begin{abstract}
The purpose of this study was to quantify ginning efficiency and fiber quality for regional cotton cultivars ginned on commercial style equipment. Results should allow plant breeders and ginners to consider improvements in cotton cultivars that are in response to ginning conditions that more nearly reflect commercial ginning practices. Therefore, the seed cotton of Giza 88 and Giza 87 growing in [Kafr El-Sheikh - Kafr El-Dawar - Etay ElBarood - Damietta], Giza 86 and Giza 85 growing in [El-Sharkia - ElGharbiya - El-Dakahliya - El-Monofiya], Giza 80 and Giza 90 growing in [Sohag - El-Minia - Beni-Sueif] were used in this study. The results obtained could be summarized as follows:

1. Growing Giza 80 and Giza 90 in Beni-Sueif location exhibited the highest gin-stand capacity, which could be explained by the environmental conditions affected fiber properties and reduced the fiber strength, fiber maturity, fiber fineness expressed as maicronaire value and that led to make ginning more easily and increased gin stand capacity.

2. No significant differences in ginning efficiency and fiber properties due to growing Giza 86 and Giza 85 in El-Sharkia, El-Gharbiya, El-Monofiya and El-Dakahliya. This could be attributed to the growing locations had the same environmental conditions (nearly the same latitude).

3. Giza 88 exhibited the highest gin-stand capacity in Kafr El-Dawar growing location, while exhibited the lowest gin-stand capacity in Etay El-Barood growing location. Also, Giza 87 exhibited the highest gin-stand capacity in Kafr El-Dawar growing location, while exhibited the lowest gin-stand capacity in Damietta growing location. This could be attributed to the difference in cotton cultivar behavior and growing the cotton cultivar in different location led to increasing the environmental variations, consequently increasing the effect of plant location on cotton characteristics.

4. It is quite clear from the results that the fiber maturity and fiber fineness expressed as maicronaire value are the most fiber properties affecting gin stand capacity with lint grade, fiber length and fiber strength.
\end{abstract}

\section{INTRODUCTION}

Ginning of cotton cultivars on conventional ginning equipment produces commercially useful information for comparative purposes. The quality of cotton fiber depends upon a number of factors ranged from seeds and sowing season to picking and other agro-climatic conditions of growth, in addition to post harvest technology employed (ginning, mill processing, etc). The work practices at ginning, storage and transportation before and after ginning also influence the quality of cotton. Hence the quality of a bale of cotton is not the same even if the variety is same. Both variety 
(genotype) and weather (growth-environment components) are accepted as important factors in determining cotton fiber quality. Each growth environment is a distinct composite of factors that can be controlled by the cotton producer (fertilization, planting date, irrigation) and uncontrolled weather factors (temperature, rainfall, and insulation). This 'quality' composite of the growth environment determines cotton fiber properties, both through modifications of metabolic rates during fiber development and through interactions between genotype and growth environment that limit realization of full genetic potential (Bradow, et al., 1996). The differential response of a genotype or cultivar across environments is defined as the genotype $x$ environment interaction ( $\mathrm{G} \times \mathrm{E}) . \mathrm{G} \times \mathrm{E}$ has long been an important and essential component of plant breeding programs dedicated to cultivar development. Bilbro and Ray (1976) indicated that a successful breeding program should focus efforts on genotype yield level (average yield compared to standards), adaptation (what environment does the genotype best perform in), and stability (how consistent does the genotype yield compared to others). When identifying improved genotypes or cultivars, plant breeders routinely practice selection (directly or indirectly) for genotypes that display stability for a given trait or set of traits across testing environments. Eberhart and Russell (1966) defined stability as the ability to show a minimum interaction with the environment. Hence, the stability of genotype performance is directly related to the effect of G x E. In cotton (Gossypium spp.) cultivar development programs, genotype stability for agronomic performance and fiber quality is an important breeding objective (Geng et al., 1987). Shalaby et al. (1993) did not found significant differences in cotton characteristics due to growing in close locations. Bassett and Kerby (1996) they stated that the variation associated with varieties was generally less than the variation associated with locations. Kerby et al. (2000) demonstrated that environment was a major factor in fiber length, fiber strength, and micronaire. Cotton breeders seek to develop genetic materials that will exceed the performance of current varieties. Agronomist conduct studies to identify the environment that optimizes cotton production. Questions have been raised regarding varietal performance and what percentage of variation in fiber quality can be accounted for as due to factors associated with varieties or with environments.

Thus, the objectives of the current study are to evaluate the effect of genotype or cultivar across environments on ginning efficiency and fiber quality, and to describe how cotton responds to various environments. Results should allow plant breeders and ginners to consider improvements in cotton cultivars that are in response to ginning conditions that more nearly reflect commercial ginning practices. 


\section{MATERIALS AND METHODS}

The present study was conducted to determine ginning efficiency and fiber quality for regional cotton cultivars ginned on commercial style equipment. The varieties and locations are listed in the following table:

\begin{tabular}{|c|c|}
\hline Cotton cultivar & location \\
\hline Giza 88 & Kafr El-Sheikh \\
Giza 87 & Kafr El-Dawar \\
& Etay El-Barood \\
& Damietta \\
\hline Giza 86 & El-Sharkia \\
Giza 85 & El-Gharbiya \\
& El-Dakahliya \\
& El-Monofiya \\
\hline Giza 80 & Sohag \\
Giza 90 & El-Minia \\
& Beni-Sueif \\
\hline
\end{tabular}

The cotton varieties used in the experiment were obtained from the 2007 growing season. The ginning performance was carried out in 2008 at the Cotton Ginning Research Section, Cotton Research Institute, Agricultural Research Center. A bulk sample for each seed-cotton variety was taken at random and elaborately mixed. For each treatment, three replications each of $5 \mathrm{~kg}$ of seed cotton were ginned by the 40-inch reciprocating knife roller gin stand (McCarthy). The ginning time of each sample was measured by a stop watch and the ginned lint was weighed. Gin stand capacity was determined and calculated according to the following equation:

\section{- Gin stand capacity $(\mathrm{kg} / \mathrm{m} \mathrm{h})=\frac{\text { Lint mass }(\mathrm{kg})}{\text { Length of roller }(\mathrm{m})} \times \frac{60}{\mathrm{GT}(\mathrm{min})}$}

Where: GT: ginning time. Length of roller: $1.01 \mathrm{~m}$ (40 in.).

The lint percentage was calculated for each sample by dividing the weight of the ginned lint by the initial seed cotton weight. A sample of 100 seeds was taken at random for each treatment, examined and repeated six times in order to get the average of cracked seed for each treatment. 
Fiber properties were tested at the Cotton Technology Research Division under standard atmospheric conditions of $(65 \% \pm 2)$ relative humidity and $\left(70^{\circ} \mathrm{F} \pm 2\right.$, $21.1^{\circ} \mathrm{C} \pm 1$ ) temperature degree. Seed cotton grades and lint grades were determined by qualified lint classers. Lint color (reflectance $\mathrm{Rd} \%$ and yellowness $+b$ ) was measured by using the "HVI colorimeter 900" according to ASTM designation D-225367 (1984). Percentage of non-lint content was measured by "microdust and trash analyzer" according to ASTM (D-2812-95). The digital fibrograph 630 instrument was operated according to ASTM (D-1447-67) to estimate the fiber length parameters [upper half mean length (mm), uniformity index (\%), and short fiber index (\%)]. Fiber fineness and maturity (micronaire value, maturity ratio and fineness) were tested by Micromat (ASTM, D - $1448-59,1984$ ). Fiber strength at $1 / 8$ inch gauge length ( $\mathrm{g} /$ tex) and fiber elongation (\%) were measured on the Stelometer according to ASTM (D-1445-75, 1984).

A factorial analysis of variance, in a completely randomized design was conducted and the differences between means were tested by Duncan's new multiple range test according to (SAS, 1996).

\section{RESULTS AND DISCUSSION}

The present investigation was conducted to determine to what degree environment (growing location), cotton cultivar and their interaction affect ginning efficiency and fiber properties. The results of ginning efficiency and fiber properties as related to cotton cultivar and growing location as well as their interactions are shown in Tables (1, 2, 3 and 4). The results show significant differences in cotton characteristics due to the effect of cotton cultivar and growing location. This could be attributed to growing the cotton cultivar in different location led to increasing the environmental variations, consequently increasing the effect of growing location on cotton characteristics.

\section{Upper-Egypt Cottons}

From the data recorded in tables 1, 2, 3 and 4, it is shown that, the cotton cultivar Giza 80 significantly exhibited higher gin stand capacity, upper half mean length, fiber yellowness, fiber strength, fiber fineness and micronaire value as compared to the cotton cultivar Giza 90, which exhibited higher color brightness and fiber elongation, while no significant differences were found between the cotton cultivar Giza 80 and Giza 90 in lint percentage, cracked seed, lint grade index, non-lint content, length uniformity index, short fiber percentage and maturity ratio.

On the other hand, the growing location Beni-Sueif exhibited the highest gin stand capacity, upper half mean length, short fiber percentage and micronaire value, 
and the lowest non-lint content and fiber fineness, while no significant differences were found between the three locations in lint percentage, cracked seed, lint grade index, uniformity index, fiber brightness, fiber yellowness, fiber strength, fiber elongation and maturity ratio. This could be attributed to the variation in environmental conditions of the growing locations (different latitude).

However, growing Giza 80 and Giza 90 in Beni-Sueif location exhibited the highest gin-stand capacity, which could be explained by the environmental conditions affected fiber properties and reduced the fiber strength, fiber maturity, fiber fineness expressed as maicronaire value and that led to make ginning more easily and increased gin stand capacity. Data indicate that fiber strength, fiber maturity, fiber fineness and maicronaire value affect gin stand capacity more than fiber length.

\section{South and Middle-Delta Cottons}

Results in tables 1, 2, 3 and 4 show that the cotton cultivar Giza 86 significantly exhibited higher lint percentage, upper half mean length, uniformity index, color brightness, fiber strength, fiber fineness and micronaire value as compared to the cotton cultivar Giza 85, which exhibited higher gin stand capacity and fiber elongation, while no significant differences were found between the cotton cultivar Giza 86 and Giza 85 in cracked seed, lint grade index, non-lint content, short fiber percentage, fiber yellowness and maturity ratio.

The results didn't show significant differences in gin stand capacity, upper half mean length, fiber strength and fiber elongation due to growing location. This could be attributed to the growing locations had the same environmental conditions (nearly the same latitude). While the small differences in environmental conditions influenced some of the properties without the other, affected lint percentage, cracked seed, uniformity index and short fiber percentage as a result of its small effect on micronaire value, maturity ratio and fiber fineness, and affect lint grade index through its effect on cracked seed, non-lint content by weight, fiber brightness and fiber yellowness.

From Tables 3 and 4, no trend was found between gin-stand capacity and fiber properties as a result of growing Giza 86 and Giza 85 in El-Sharkia, El-Gharbiya, ElMonofiya and El-Dakahliya. This could be attributed to the cotton cultivars Giza 86 and Giza 85 have nearly the same behavior in the four growing locations. Giza 86 exhibited the highest gin-stand capacity in El-Monofiya growing location at the highest micronaire value and high upper half mean length and fiber strength, while exhibited the lowest gin-stand capacity in El-Dakahliya growing location at the lowest micronaire value and high upper half mean length and fiber strength. While Giza 85 exhibited the highest gin-stand capacity in El-Gharbiya growing location at the lowest micronaire value and fiber strength and high upper half mean length, while exhibited the lowest 
gin-stand capacity in El-Monofiya growing location at the highest micronaire value and high upper half mean length and fiber strength. It is quite clear from the results that the fiber strength, fiber maturity, fiber fineness and maicronaire value with lint grade and fiber length are the most fiber properties affecting gin stand capacity.

\section{North-Delta Cottons}

Results in tables 1, 2, 3 and 4 observed that the cotton cultivar Giza 88 significantly exhibited the highest gin-stand capacity, lint percentage, lint grade index, fiber yellowness and micronaire value as compared to the cotton cultivar Giza 87 one, which exhibited the highest fiber brightness, maturity ratio and fiber fineness, while no significant differences were found between the cotton cultivar Giza 88 and Giza 87 in cracked seed, non-lint content, upper half mean length, short fiber percentage and fiber elongation.

The growing location Damietta exhibited the lowest gin stand capacity, lint percentage and micronaire value, and the highest lint grade index, uniformity index, fiber brightness, fiber yellowness, fiber strength and maturity ratio, while no significant differences were found between the four locations in cracked seed, non-lint content, upper half mean length, short fiber percentage, fiber elongation and fiber fineness. However, The growing location Kafr El-Dawar exhibited the highest gin stand capacity, lint percentage, lint grade index, uniformity index, fiber brightness, fiber yellowness, fiber strength, micronaire value and maturity ratio. This could be attributed to the variation in environmental conditions of the growing locations (different latitude).

Also, it could be noticed that Giza 88 exhibited the highest gin-stand capacity in Kafr El-Dawar growing location at the highest upper half mean length, fiber strength and micronaire value, while exhibited the lowest gin-stand capacity in Etay El-Barood growing location at the lowest upper half mean length, fiber strength and micronaire value. But Giza 87 exhibited the highest gin-stand capacity in Kafr El-Dawar growing location at the highest upper half mean length and micronaire value and the howest fiber strength, while exhibited the lowest gin-stand capacity in Damietta growing location at the highest upper half mean length and fiber strength and the lowest micronaire value. 
Table 1. Ginning efficiency as affected by the cotton variety and growing location.

\begin{tabular}{|c|c|c|c|c|c|c|}
\hline & Treatment & 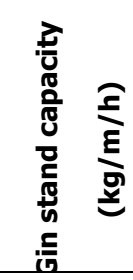 & 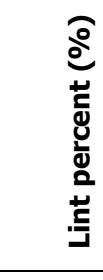 & 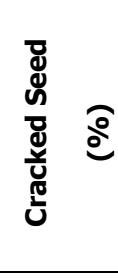 & 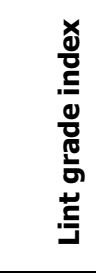 & 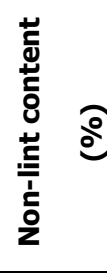 \\
\hline \multicolumn{7}{|c|}{ Upper-Egypt Cottons } \\
\hline \multirow[t]{2}{*}{ Variety: } & Giza 80 & $42.1 \mathrm{a}$ & $37.1 \mathrm{a}$ & 2.3a & $41.9 \mathrm{a}$ & $6.0 \mathrm{a}$ \\
\hline & Giza 90 & $39.2 b$ & $36.0 \mathrm{a}$ & $2.0 \mathrm{a}$ & $42.1 \mathrm{a}$ & $5.5 \mathrm{a}$ \\
\hline \multirow[t]{4}{*}{ Location: } & Sohag & $40.0 \mathrm{~b}$ & $35.6 a$ & $2.2 \mathrm{a}$ & $41.8 \mathrm{a}$ & $5.8 \mathrm{a}$ \\
\hline & El-Minia & $38.9 \mathrm{~b}$ & $37.0 \mathrm{a}$ & $2.2 \mathrm{a}$ & $41.8 \mathrm{a}$ & 7.1a \\
\hline & Beni-Sueif & $43.0 \mathrm{a}$ & $37.1 \mathrm{a}$ & $2.2 \mathrm{a}$ & 42.3a & $4.3 \mathrm{~b}$ \\
\hline & Mean & 40.65 & 36.5 & 2.2 & 42 & 5.7 \\
\hline \multicolumn{7}{|c|}{ South and Middle-Delta Cottons } \\
\hline \multirow[t]{2}{*}{ Variety: } & Giza 85 & $45.4 a$ & $36.4 \mathrm{~b}$ & $1.6 \mathrm{a}$ & $43.3 a$ & $3.7 a$ \\
\hline & Giza 86 & $44.1 \mathrm{~b}$ & $37.1 \mathrm{a}$ & $2.5 \mathrm{a}$ & $43.5 \mathrm{a}$ & 4.0a \\
\hline \multirow[t]{5}{*}{ Location: } & El-Sharkia & $44.7 \mathrm{a}$ & $36.6 \mathrm{~b}$ & $2.8 \mathrm{a}$ & $43.0 \mathrm{~b}$ & 4.6a \\
\hline & El-Gharbiya & 44.9a & $36.8 \mathrm{ab}$ & $1.7 \mathrm{ab}$ & $43.8 \mathrm{a}$ & 4.1a \\
\hline & El-Monofiya & $45.5 \mathrm{a}$ & $36.5 \mathrm{~b}$ & $1.2 \mathrm{~b}$ & 43.3ab & 4.1a \\
\hline & El-Dakahliya & $43.9 \mathrm{a}$ & $37.3 a$ & $2.5 \mathrm{ab}$ & $43.5 \mathrm{ab}$ & $2.8 \mathrm{~b}$ \\
\hline & Mean & 44.8 & 36.8 & 2.0 & 43.4 & 3.9 \\
\hline \multicolumn{7}{|c|}{ North-Delta Cottons } \\
\hline \multirow[t]{2}{*}{ Variety: } & Giza 87 & $43.3 \mathrm{~b}$ & $32.7 \mathrm{~b}$ & $1.1 \mathrm{a}$ & $41.6 \mathrm{~b}$ & $5.4 a$ \\
\hline & Giza 88 & $46.1 \mathrm{a}$ & $37.3 a$ & $2.4 a$ & $42.2 \mathrm{a}$ & $4.8 \mathrm{a}$ \\
\hline \multirow[t]{5}{*}{ Location: } & Kafr El-Sheikh & $45.8 \mathrm{ab}$ & $35.6 a$ & $1.7 a$ & $41.7 a b$ & $6.0 \mathrm{a}$ \\
\hline & Kafr El-Dawar & $47.9 \mathrm{a}$ & $35.3 a$ & $2.2 \mathrm{a}$ & $42.2 \mathrm{a}$ & $5.0 \mathrm{a}$ \\
\hline & Etay El-Barood & $43.2 \mathrm{bc}$ & $34.7 \mathrm{~b}$ & $1.2 \mathrm{a}$ & $41.5 \mathrm{~b}$ & $4.5 \mathrm{a}$ \\
\hline & Damietta & $41.7 \mathrm{c}$ & $34.4 \mathrm{~b}$ & $2.0 \mathrm{a}$ & $42.2 \mathrm{a}$ & $4.9 \mathrm{a}$ \\
\hline & Mean & 44.67 & 35.00 & 1.75 & 41.88 & 5.10 \\
\hline
\end{tabular}


Table 2. Fiber properties as affected by the cotton variety and growing location.

\begin{tabular}{|c|c|c|c|c|c|c|c|c|c|c|c|}
\hline \multirow{2}{*}{\multicolumn{2}{|c|}{ Treatment }} & \multicolumn{3}{|c|}{$\begin{array}{c}\text { Fiber length } \\
\text { parameter }\end{array}$} & \multicolumn{2}{|c|}{ Lint color } & \multicolumn{2}{|c|}{$\begin{array}{c}\text { Fiber } \\
\text { tenacity }\end{array}$} & \multicolumn{3}{|c|}{$\begin{array}{c}\text { Fiber fineness \& } \\
\text { maturity }\end{array}$} \\
\hline & & 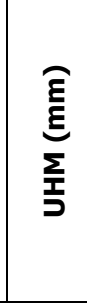 & 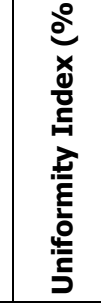 & 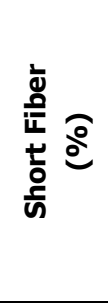 & 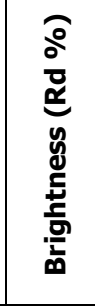 & 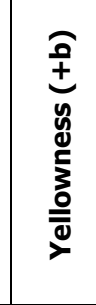 & 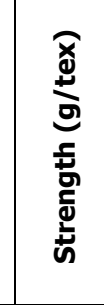 & 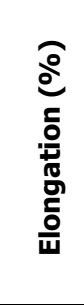 & 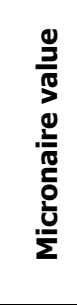 & 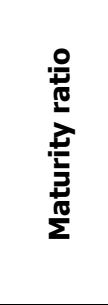 & 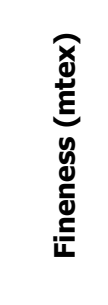 \\
\hline \multicolumn{12}{|c|}{ Upper-Egypt Cottons } \\
\hline \multirow[t]{2}{*}{ Variety: } & Giza 80 & $31.0 \mathrm{a}$ & $85.0 a$ & $8.0 \mathrm{a}$ & $61.2 b$ & $13.7 a$ & $38.9 a$ & $7.2 \mathrm{~b}$ & $4.2 a$ & $0.88 a$ & $177.0 \mathrm{a}$ \\
\hline & Giza 90 & $28.9 b$ & $84.2 a$ & $8.0 a$ & 65.0a & $13.0 \mathrm{~b}$ & $36.7 \mathrm{~b}$ & $7.8 \mathrm{a}$ & $4.0 \mathrm{~b}$ & $0.89 a$ & $163.6 \mathrm{~b}$ \\
\hline \multirow[t]{4}{*}{ Location: } & Sohag & $30.7 a$ & 85.0a & $7.4 \mathrm{~b}$ & 61.9a & 13.5a & $37.6 a$ & $7.4 a$ & $4.2 \mathrm{~b}$ & $0.89 a$ & $170.4 a b$ \\
\hline & El-Minia & $28.8 b$ & $84.0 \mathrm{a}$ & $8.5 a$ & $62.8 a$ & $13.3 a$ & $38.6 a$ & 7.3a & $3.7 c$ & $0.85 a$ & $157.2 b$ \\
\hline & $\begin{array}{l}\text { Beni- } \\
\text { Sueif }\end{array}$ & $30.4 a$ & $84.7 a$ & $8.0 a b$ & $64.6 a$ & $13.4 a$ & $37.2 a$ & $7.8 \mathrm{a}$ & $4.4 a$ & $0.92 a$ & $183.2 \mathrm{a}$ \\
\hline & Mean & 29.9 & 84.6 & 8.0 & 63.1 & 13.4 & 37.8 & 7.5 & 4.1 & 0.89 & 170.3 \\
\hline \multicolumn{12}{|c|}{ South and Middle-Delta Cottons } \\
\hline \multirow[t]{2}{*}{ Variety: } & Giza 85 & $31.1 \mathrm{~b}$ & $85.8 \mathrm{~b}$ & $7.0 \mathrm{a}$ & $71.8 \mathrm{~b}$ & $10.2 a$ & $38.3 b$ & $7.4 a$ & $4.3 b$ & $1.02 \mathrm{a}$ & $162.0 \mathrm{~b}$ \\
\hline & Giza 86 & $33.7 a$ & $87.2 \mathrm{a}$ & $7.0 \mathrm{a}$ & $73.5 a$ & 10.1a & $40.7 a$ & $5.9 b$ & $4.7 a$ & $1.06 a$ & $175.0 \mathrm{a}$ \\
\hline \multirow[t]{5}{*}{ Location: } & $\begin{array}{c}\text { El- } \\
\text { Sharkia }\end{array}$ & $31.8 \mathrm{a}$ & $85.3 b$ & $7.4 a$ & $72.3 b$ & $10.1 \mathrm{bc}$ & $38.6 a$ & $7.0 \mathrm{a}$ & $4.4 \mathrm{~b}$ & $1.02 \mathrm{ab}$ & $168.0 \mathrm{~b}$ \\
\hline & $\begin{array}{c}\text { El- } \\
\text { Gharbiya }\end{array}$ & $32.5 a$ & $86.8 \mathrm{a}$ & $6.9 a b$ & $72.4 b$ & $10.2 a b$ & $39.1 a$ & $6.8 \mathrm{a}$ & $4.5 b$ & $1.11 \mathrm{a}$ & $159.2 b$ \\
\hline & $\begin{array}{c}\text { El- } \\
\text { Monofiya }\end{array}$ & $32.6 \mathrm{a}$ & $87.2 a$ & $6.9 a b$ & $70.9 b$ & $10.4 a$ & $40.3 a$ & $6.6 a$ & $4.7 a$ & $0.99 b$ & $185.5 a$ \\
\hline & $\begin{array}{c}\text { El- } \\
\text { Dakahliya }\end{array}$ & $32.7 \mathrm{a}$ & $86.8 \mathrm{a}$ & $6.7 b$ & $74.9 a$ & $9.9 \mathrm{c}$ & $40.0 a$ & $6.3 a$ & $4.3 b$ & $1.04 a b$ & $161.3 b$ \\
\hline & Mean & 32.4 & 86.5 & 7.0 & 72.6 & 10.1 & 39.5 & 6.7 & 4.5 & 1.04 & 168.5 \\
\hline \multicolumn{12}{|c|}{ North-Delta Cottons } \\
\hline \multirow[t]{2}{*}{ Variety: } & Giza 87 & $34.7 a$ & 87.5a & $7.4 a$ & $68.3 a$ & $11.3 \mathrm{~b}$ & $42.5 a$ & $5.8 a$ & $3.3 b$ & $1.00 \mathrm{a}$ & $120.7 b$ \\
\hline & Giza 88 & $35.1 \mathrm{a}$ & 87.6a & $7.4 a$ & $63.5 b$ & $13.5 a$ & $43.4 a$ & $5.5 a$ & $4.0 \mathrm{a}$ & $0.93 b$ & $163.4 a$ \\
\hline \multirow[t]{5}{*}{ Location: } & $\begin{array}{c}\text { Kafr El- } \\
\text { Sheikh } \\
\end{array}$ & $35.0 a$ & 87.7ab & 7.5a & $66.4 a$ & $11.9 \mathrm{~b}$ & $43.8 a$ & $5.7 a$ & $3.9 a$ & $1.01 \mathrm{a}$ & $145.9 a$ \\
\hline & $\begin{array}{l}\text { Kafr El- } \\
\text { Dawar }\end{array}$ & $35.0 a$ & $87.6 a b$ & $7.4 a$ & 65.9ab & $12.6 a$ & $42.6 a b$ & $5.7 a$ & $3.9 a$ & $1.03 a$ & $146.4 a$ \\
\hline & $\begin{array}{l}\text { Etay El- } \\
\text { Barood }\end{array}$ & $34.6 a$ & 87.0b & $7.4 a$ & $64.4 b$ & $12.7 a$ & $40.8 b$ & $5.4 a$ & $3.5 b$ & $0.88 b$ & $139.8 \mathrm{a}$ \\
\hline & Damietta & $35.1 a$ & 88.0a & $7.2 a$ & 67.0a & $12.4 a$ & $44.7 a$ & $5.8 a$ & $3.5 b$ & $0.95 a b$ & $136.1 \mathrm{a}$ \\
\hline & Mean & 34.92 & 87.58 & 7.37 & 65.92 & 12.41 & 42.96 & 5.64 & 3.7 & 0.97 & 142.1 \\
\hline
\end{tabular}


Table 3. Mean values of ginning characteristics as affected by the cotton variety and growing location.

\begin{tabular}{|c|c|c|c|c|c|c|}
\hline $\begin{array}{l}\frac{7}{0} \\
\frac{20}{\pi} \\
>\end{array}$ & . & 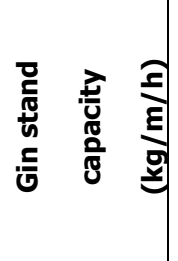 & 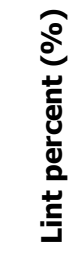 & 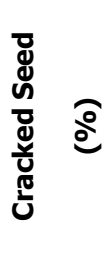 & 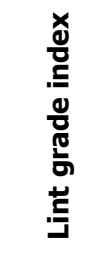 & 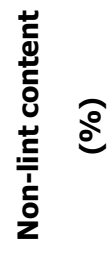 \\
\hline \multicolumn{7}{|c|}{ Upper-Egypt Cottons } \\
\hline \multirow[t]{3}{*}{ Giza 80} & Sohag & 42.8 & 35.8 & 2.7 & 41.7 & 6.9 \\
\hline & El-Minia & 40.2 & 37.6 & 2.0 & 41.7 & 7.3 \\
\hline & Beni-Sueif & 43.2 & 37.9 & 2.3 & 42.3 & 3.6 \\
\hline \multirow[t]{3}{*}{ Giza 90} & Sohag & 37.2 & 35.3 & 1.7 & 42.0 & 4.7 \\
\hline & El-Minia & 37.6 & 36.3 & 2.3 & 42.0 & 6.9 \\
\hline & Beni-Sueif & 42.9 & 36.2 & 2.0 & 42.3 & 4.9 \\
\hline \multicolumn{7}{|c|}{ South and Middle-Delta Cottons } \\
\hline \multirow[t]{4}{*}{ Giza 85} & El-Sharkia & 44.9 & 35.6 & 3.0 & 42.3 & 4.7 \\
\hline & El-Gharbiya & 46.4 & 36.1 & 1.3 & 43.7 & 4.2 \\
\hline & El-Monofiya & 44.8 & 36.3 & 1.3 & 43.3 & 3.6 \\
\hline & El-Dakahliya & 45.4 & 37.7 & 0.7 & 44.0 & 2.5 \\
\hline \multirow[t]{4}{*}{ Giza 86} & El-Sharkia & 44.4 & 37.6 & 2.7 & 43.7 & 4.5 \\
\hline & El-Gharbiya & 43.4 & 37.5 & 2.0 & 44.0 & 4.0 \\
\hline & El-Monofiya & 46.3 & 36.6 & 1.0 & 43.3 & 4.6 \\
\hline & El-Dakahliya & 42.5 & 37.0 & 4.3 & 43.0 & 3.0 \\
\hline \multicolumn{7}{|c|}{ North-Delta Cottons } \\
\hline \multirow[t]{4}{*}{ Giza 87} & Kafr El-Sheikh & 45.5 & 33.1 & 0.7 & 41.0 & 7.0 \\
\hline & Kafr El-Dawar & 46.9 & 32.8 & 1.7 & 42.0 & 4.3 \\
\hline & Etay El-Barood & 42.0 & 32.5 & 0.7 & 41.0 & 5.0 \\
\hline & Damietta & 38.8 & 32.4 & 1.3 & 42.3 & 5.4 \\
\hline \multirow[t]{4}{*}{ Giza 88} & Kafr El-Sheikh & 46.2 & 38.1 & 2.7 & 42.3 & 5.0 \\
\hline & Kafr El-Dawar & 48.9 & 37.8 & 2.7 & 42.3 & 5.7 \\
\hline & Etay El-Barood & 44.4 & 37.0 & 1.7 & 42.0 & 4.0 \\
\hline & Damietta & 44.7 & 36.4 & 2.7 & 42.0 & 4.4 \\
\hline
\end{tabular}


Table 4. Mean values of fiber characteristics as affected by the cotton variety and growing location.

\begin{tabular}{|c|c|c|c|c|c|c|c|c|c|c|c|}
\hline \multirow[b]{2}{*}{ 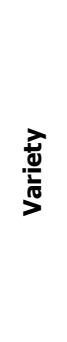 } & \multirow[b]{2}{*}{ 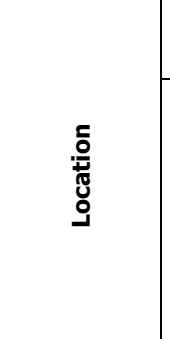 } & \multicolumn{3}{|c|}{ Fiber length parameter } & \multicolumn{2}{|c|}{ Lint color } & \multicolumn{2}{|c|}{$\begin{array}{c}\text { Fiber } \\
\text { tenacity }\end{array}$} & \multicolumn{3}{|c|}{$\begin{array}{c}\text { Fiber fineness \& } \\
\text { maturity }\end{array}$} \\
\hline & & 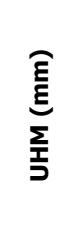 & 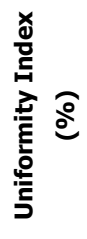 & 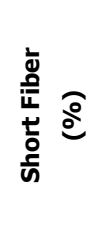 & 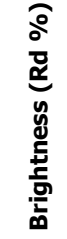 & 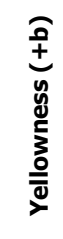 & 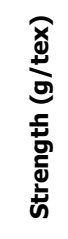 & 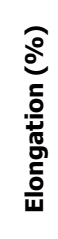 & 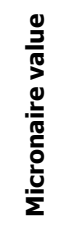 & 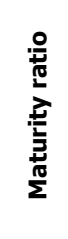 & 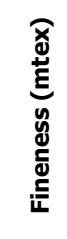 \\
\hline \multicolumn{12}{|c|}{ Upper-Egypt Cottons } \\
\hline \multirow[t]{3}{*}{$\begin{array}{c}\text { Giza } \\
80 \\
\end{array}$} & Sohag & 31.4 & 84.5 & 7.4 & 60.5 & 13.9 & 38.5 & 7.5 & 4.2 & 0.90 & 165.7 \\
\hline & El-Minia & 29.9 & 84.7 & 8.1 & 61.7 & 13.6 & 40.1 & 6.7 & 3.7 & 0.83 & 160.6 \\
\hline & Beni-Sueif & 31.6 & 85.8 & 8.4 & 61.4 & 13.6 & 38.0 & 7.3 & 4.7 & 0.89 & 204.6 \\
\hline \multirow[t]{3}{*}{$\begin{array}{c}\text { Giza } \\
90 \\
\end{array}$} & Sohag & 30.0 & 85.5 & 7.3 & 63.3 & 12.9 & 36.7 & 7.4 & 4.1 & 0.87 & 175.0 \\
\hline & El-Minia & 27.6 & 83.4 & 9.0 & 63.9 & 12.9 & 37.2 & 7.8 & 3.7 & 0.86 & 153.9 \\
\hline & Beni-Sueif & 29.1 & 83.6 & 7.5 & 67.7 & 13.2 & 36.3 & 8.2 & 4.1 & 0.95 & 161.8 \\
\hline \multicolumn{12}{|c|}{ South and Middle-Delta Cottons } \\
\hline \multirow[t]{4}{*}{$\begin{array}{c}\text { Giza } \\
85 \\
\end{array}$} & El-Sharkia & 31.3 & 85.0 & 7.1 & 71.1 & 10.4 & 36.5 & 7.8 & 4.2 & 1.0 & 155.1 \\
\hline & El-Gharbiya & 31.0 & 85.8 & 7.0 & 70.4 & 10.1 & 36.8 & 7.1 & 4.2 & 1.0 & 158.0 \\
\hline & El-Monofiya & 31.2 & 86.3 & 7.1 & 69.5 & 10.3 & 39.7 & 7.6 & 4.4 & 1.1 & 164.7 \\
\hline & El-Dakahliya & 31.0 & 86.3 & 6.7 & 76.2 & 9.9 & 40.4 & 7.2 & 4.3 & 1.0 & 170.2 \\
\hline \multirow[t]{4}{*}{$\begin{array}{c}\text { Giza } \\
86 \\
\end{array}$} & El-Sharkia & 32.4 & 85.5 & 7.6 & 73.5 & 9.7 & 40.7 & 6.1 & 4.6 & 1.0 & 180.8 \\
\hline & El-Gharbiya & 34.0 & 87.9 & 6.9 & 74.4 & 10.3 & 41.4 & 6.5 & 4.7 & 1.2 & 160.5 \\
\hline & El-Monofiya & 34.1 & 88.1 & 6.7 & 72.3 & 10.5 & 40.9 & 5.7 & 4.9 & 0.9 & 206.4 \\
\hline & El-Dakahliya & 34.4 & 87.3 & 6.6 & 73.6 & 9.8 & 39.7 & 5.3 & 4.3 & 1.1 & 152.5 \\
\hline \multicolumn{12}{|c|}{ North-Delta Cottons } \\
\hline \multirow[t]{4}{*}{$\begin{array}{c}\text { Giza } \\
87 \\
\end{array}$} & $\begin{array}{l}\text { Kafr El- } \\
\text { Sheikh }\end{array}$ & 34.6 & 87.4 & 7.5 & 68.8 & 10.7 & 43.8 & 6.0 & 3.5 & 1.09 & 118.2 \\
\hline & $\begin{array}{l}\text { Kafr El- } \\
\text { Dawar }\end{array}$ & 34.9 & 87.5 & 7.4 & 69.0 & 11.5 & 40.5 & 6.0 & 3.6 & 1.09 & 121.8 \\
\hline & $\begin{array}{l}\text { Etay El- } \\
\text { Barood }\end{array}$ & 34.8 & 87.4 & 7.3 & 65.6 & 11.7 & 40.1 & 5.5 & 3.1 & 0.89 & 123.2 \\
\hline & Damietta & 34.5 & 87.9 & 7.3 & 69.9 & 11.2 & 45.5 & 5.6 & 3.2 & 0.96 & 119.5 \\
\hline \multirow[t]{4}{*}{$\begin{array}{c}\text { Giza } \\
88 \\
\end{array}$} & Kafr El-Shei & 35.4 & 88.1 & 7.6 & 63.9 & 13.2 & 43.8 & 5.3 & 4.3 & 0.93 & 173.5 \\
\hline & $\begin{array}{l}\text { Kafr El- } \\
\text { Dawar }\end{array}$ & 35.0 & 87.6 & 7.4 & 62.8 & 13.6 & 44.7 & 5.5 & 4.3 & 0.97 & 171.0 \\
\hline & $\begin{array}{l}\text { Etay El- } \\
\text { Barood }\end{array}$ & 34.4 & 86.7 & 7.5 & 63.2 & 13.7 & 41.4 & 5.3 & 3.8 & 0.88 & 156.5 \\
\hline & Damietta & 35.7 & 88.1 & 7.0 & 64.1 & 13.6 & 43.9 & 5.9 & 3.8 & 0.94 & 152.8 \\
\hline
\end{tabular}




\section{REFERENCES}

1. ASTM. 1984. D-2253-67, D-1447-67, D-1445-75, D-1448-59 and D-2812-95. Philadelphia, Pa.: U.S.A.

2. Bassett, D.M. and T.A. Kerby. 1996. Variety Selection. pp. 123 to 133. In Cotton Production Manual. (eds.) S. Johnson Hake, T.A. Kerby, and K.D. Hake. Univ. California Publication 3352.

3. Bilbro, J.D. and L.L. Ray. 1976. Environmental stability and adaptation of several cotton cultivars. Crop Sci. 16:821-824.

4. Bradow, J.M., G.H. Davidonis, O. Hinojosa, L.H. Wartelle, K.J. Pratt, K. Pustateri, P.J. Bauer, B. Fisher, G.F. Sassenrath-Cole, P.H. Dastoor, A. Johnson, J.A. Landivar, D. Locke and D. Moseley. 1996. Environmentally induced variations in cotton fiber maturity and related yarn and dyed knit defects. Proceedings Beltwide Cotton Conf., 1279-1284.

5. Eberhart, S.A. and W.A. Russell. 1966. Stability parameters for comparing varieties. Crop Sci. 6:36-40.

6. Geng, S., Q.F. Zhang and D.M. Bassett. 1987. Stability in yield and fiber quality of California cotton. Crop Sci. 27:1004-1010.

7. Kerby, Tom, Janet Burgess, Marc Bates, Dave Albers and Ken Lege. 2000. Partitioning variety and environment contribution to variation in yield, plant growth, and fiber quality. pp. 528-532. . Beltwide Cotton Conf. Nat'l Cotton Council, Memphis, TN.

8. SAS User's Guide. 1996. Statistics Ver. 5. Cary, N.C.: SAS Institute, Inc. :433-506.

9. Shalaby, A.W., M.A. Eweida and lila M.A. Abdel-Rahman. 1993. Ginning outturn and lint grade as related to cotton plant location and fiber characteristics. Egypt. J. Appl. Sci., 8(12): 967-975. 


\section{تأثير صنف القطن و منطقة زراعته على كفاءة الحلج وجودة القطن}

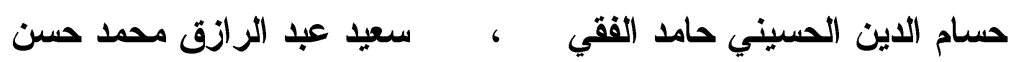

$$
\text { معة بحوث القطن - مركز البحوث الزراعية - الجبزة }
$$

أجري هذا البحث لار اسة تأثير صنف القطن ومنطقة زر اعته على كفاءة الحلج وجودة

الألباف. وقد استخدم لهذه الدراسة الصنفان: جيزة 87، وجيزة 88 نم زر اعتهما في مناطق ككفر

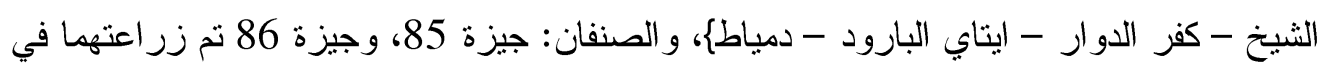

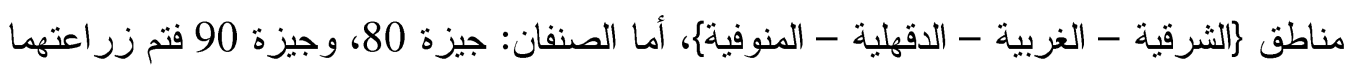
في \}سوهاج - المنيا - بني سويف\{. وقد أسفرت الدراسة عن النتائج التالية:

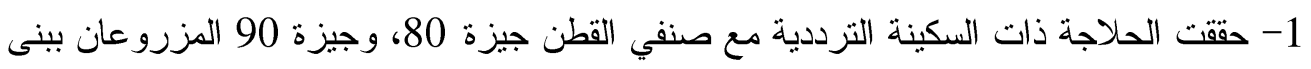

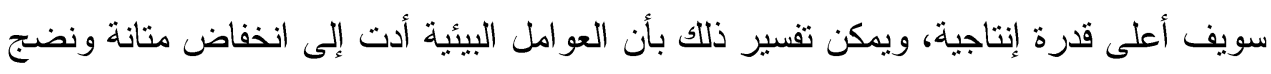

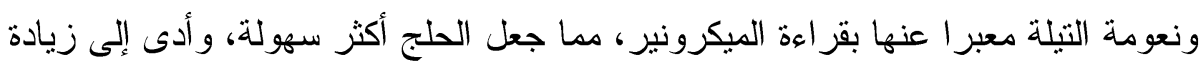
إنتاجية الحلاجة.

2- لم يُظهر صنفا القطن جيزة 86، وجيزة 85 مع مناطق الزر اعة تأثير ا معنويا على كفاءة الحلج

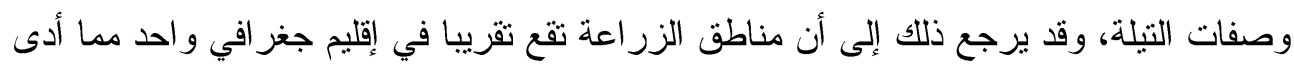

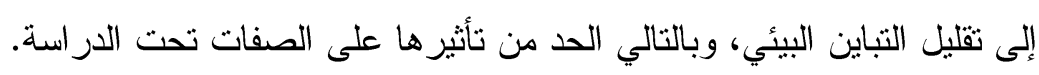

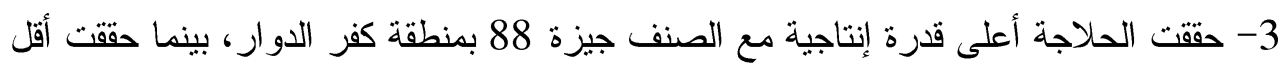
87

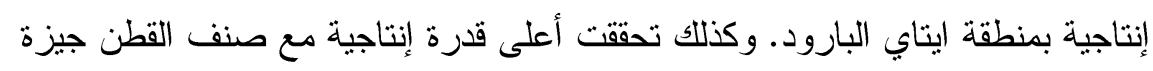

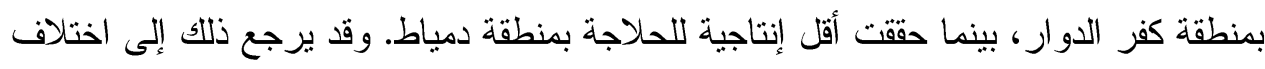

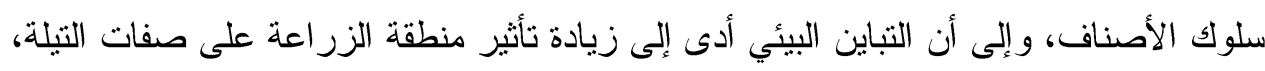

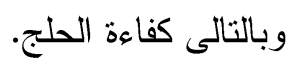
4- أوضحت النتائج أن نضج ونعومة الثعرة معبر ا عنها بقراعة الميكرونير أكثر الصفات تأثيرا

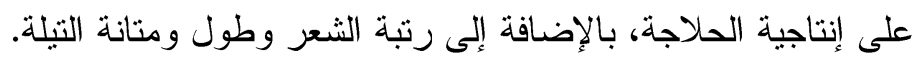

